\title{
Systems biology approaches to investigating the roles of extracellular vesicles in human diseases
}

\author{
András Gézsi ${ }^{1,2,3}$, Árpád Kovács ${ }^{1}$ Tamás Visnovitz and Edit I. Buzás ${ }^{1,2}$
}

\begin{abstract}
Extracellular vesicles (EVs) are membrane-enclosed structures secreted by cells. In the past decade, EVs have attracted substantial attention as carriers of complex intercellular information. They have been implicated in a wide variety of biological processes in health and disease. They are also considered to hold promise for future diagnostics and therapy. EVs are characterized by a previously underappreciated heterogeneity. The heterogeneity and molecular complexity of EVs necessitates high-throughput analytical platforms for detailed analysis. Recently, mass spectrometry, nextgeneration sequencing and bioinformatics tools have enabled detailed proteomic, transcriptomic, glycomic, lipidomic, metabolomic, and genomic analyses of EVs. Here, we provide an overview of systems biology experiments performed in the field of EVs. Furthermore, we provide examples of how in silico systems biology approaches can be used to identify correlations between genes involved in EV biogenesis and human diseases. Using a knowledge fusion system, we investigated whether certain groups of proteins implicated in the biogenesis/release of EVs were associated with diseases and phenotypes. Furthermore, we investigated whether these proteins were enriched in publicly available transcriptomic datasets using gene set enrichment analysis methods. We found associations between key EV biogenesis proteins and numerous diseases, which further emphasizes the key role of EVs in human health and disease.
\end{abstract}

\section{Introduction}

The rapidly emerging field of extracellular vesicles (EVs) has led to paradigm shifts in many different areas of biology and biomedicine. The release of EVs, originally thought to only act to remove harmful substances from cells, has been shown to have many more functional consequences and a wide range of implications for biomedicine. To understand the structure and function of EVs, the initial biochemical targeted approaches rapidly progressed to bias-free large-scale analyses using systems biology and bioinformatics. In 2009, the first manually curated database of EV proteins, RNA and lipids, Exo$\operatorname{Carta}^{1}$ (http://www.exocarta.org/), was launched. It was

Correspondence: Edit I. Buzás (buzas.edit@med.semmelweis-univ.hu)

'Department of Genetics, Cell- and Immunobiology, Semmelweis University, Budapest, Hungary

${ }^{2}$ MTA-SE Immune-Proteogenomics Extracellular Vesicle Research Group, Budapest, Hungary

Full list of author information is available at the end of the article. followed by two additional databases including Vesiclepedia $^{2,3}$ (http://www.microvesicles.org/) and EVpedia ${ }^{4,5}$ (http://student4.postech.ac.kr/evpedia2_xe/xe/). These are repositories of RNA, protein, lipid, and metabolite datasets. Given that preanalytical parameters may play important roles in the quality of EV preparations, database entries should be interpreted with caution, and special attention has to be paid to preanalytical conditions. Recently, gene ontology has been extended to the context of EV communication, owing to increased recognition of the importance of the EV field ${ }^{6}$. Furthermore, bioinformatic tools that can be used to analyze EV datasets have become available ${ }^{7,8}$. Future directions may include the following: (i) systems biology analyses after more standardized EV preanalytics, (ii) multiomics analyses of EV samples (combinations of different -omic groups used for the analysis), and (iii) the determination of disease-specific EV molecular patterns/networks composed of different molecule types. Additionally, systems 
biology approaches may be extended to novel fields such as image-based systems biology.

Advancements in the analysis of complex biological systems such as EVs will help to reveal the biological significance of these recently discovered structures and exploit their diagnostic and/or therapeutic potential.

\section{EV proteomics}

To date, the best characterized EV cargo is EVassociated protein molecules. Proteomics analysis of EVs has been made available on MS-based technological platforms. Proteomic analyses of EVs have been reviewed extensively elsewhere ${ }^{9,10}$ and are not the focus of the present article. Of note, thousands of proteins have been identified in various EV subtypes, and disease-specific proteome alterations have also been identified ${ }^{11-14}$. The potential for EV proteins to be used as monitoring tools for disease progression has also been successfully stu$\operatorname{died}^{15}$. In addition, unconventional membrane protein orientation has been described in $\mathrm{EVs}^{16}$. The topology of various $\mathrm{EV}$-associated proteins remains a very important hot topic because it influences target cell recognition by different EV subtypes and the signal transduction pathways induced by EVs.

\section{EV transcriptomics}

A plethora of studies confirmed the feasibility of using high-throughput transcriptomic methods for EVs (such as microarrays and next-generation sequencing; see Table 1$)^{17-19}$, and these approaches have been used successfully to characterize the healthy circulating ${ }^{20,21}$, urine $^{20,22}$, cerebrospinal fluid ${ }^{23}$, or saliva ${ }^{24,25}$ EV RNA cargo. The first study exploring the physiological miRNA pattern of circulating EVs was published in $2008^{26}$. In the following years, the heterogeneity of circulating EV transcriptional landscapes was analyzed and revealed the presence of many different RNA types, including tRNA, miRNA, Y-RNA, mRNA, SRP-RNA, rRNA, IncRNA, piRNA, snRNA, snoRNA, and scaRNA ${ }^{17,20,21}$. In vitro studies further suggested that various types of RNA molecules identified in EVs were specifically shuttled into EV subsets ${ }^{27}$. A reference dataset for miRNA profiling in whole blood, peripheral blood cells, serum, and EVs was also published ${ }^{28}$. EV transcriptomics is particularly useful in the study of complex diseases because it assists in the identification of novel biomarkers (Table 1). The biomarker potential of EVs has been highlighted by high-throughput studies; however, the analysis of a single subtype of $\mathrm{EVs}^{29}$ instead of bulk EV 'omics' analyses may yield more targeted results and suggest novel therapeutic strategies.

\section{EV metabolomics}

Metabolomics involves the simultaneous detection and analysis of a large number of small molecules $(<2000 \mathrm{Da})$ from biological samples ${ }^{30}$. The relatively low sensitivity of NMR to detect metabolites in EV samples (which are usually available in low amounts) does not allow detailed analysis of the EV metabolome. However, with advances in the available methodological platforms (e.g., UltraPerformance Liquid Chromatography-Mass Spectrometry, UPLC MS), numerous studies have performed detailed analyses of the EV metabolome ${ }^{31-34}$. Interestingly, EVs have been shown to function as independent metabolic units ${ }^{35}$ and to modify the metabolome of their body fluid environment ${ }^{36,37}$ or to induce metabolic changes in recipient cells ${ }^{38}$.

\section{EV lipidomics}

EV lipidomics (see Table 2) is a relatively new field mainly because the amount of an EV sample is usually very limited, and novel techniques with increased sensitivity have only recently become available to EV researchers. In the twentieth century and in the first decade of the twenty-first century, thin layer chromatography (TLC) was widely used, and it was essentially the only technique available that enabled the study of the lipid composition of EV membranes. TLC is an easy and straightforward method and does not require expensive equipment. However, the data collected in TLC experiments are very limited. Only a few lipid forms (main classes) can be separated with the help of external lipid standards. Since 2004, the application of different liquid chromatography technologies have been reported. The sensitivity and reproducibility of these experiments were significantly improved compared to those of the TLC methods, but the number of detectable lipid species was still very limited. Revolutionary development began in the early 2010s with MSbased methods, when real EV lipidomics began. The different MS-based techniques made it possible to determine the different acyl chains of membrane lipids (not just the major lipid types based on the head groups). The number of complex lipidomic studies started to increase significantly in 2016, and an exponential growth of the field is expected to come in the next few years.

\section{EV glycomics}

Glycomics in general show a relative backlog compared to other omic fields, such as genomics or proteomics (see Table 3). This is possibly due to the complexity of carbohydrate structures and the lack of sensitive and simple high-throughput methods for glycan analysis that caused a significant delay in the development of glycomics. For glycosylation analyses of EVs, lectin-based microarrays, and high resolution MS analyses have been used, and these approaches provide evidence of EV-specific glycosylation patterns. 


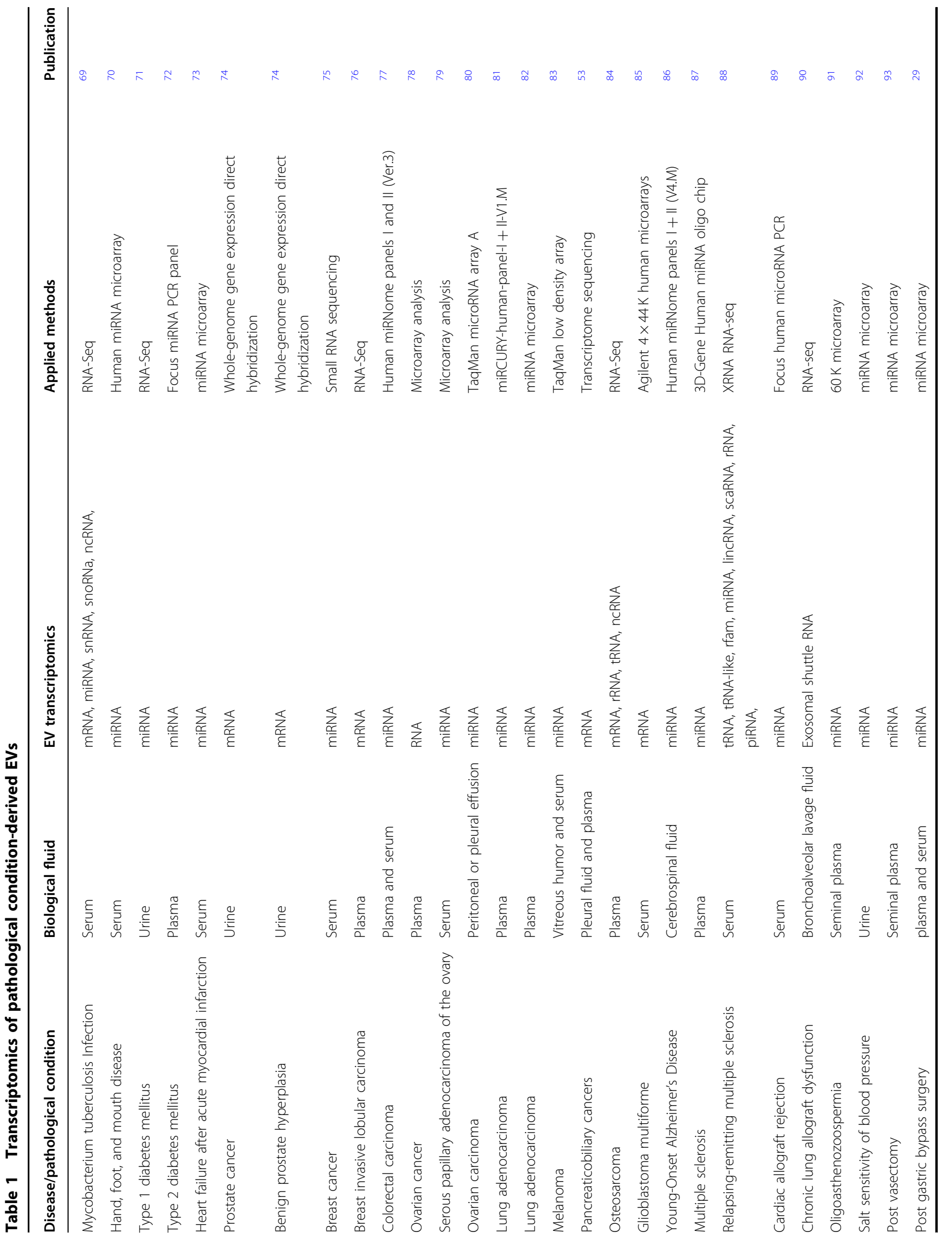


Table 2 Lipidomic analyses of EVs

\begin{tabular}{|c|c|c|}
\hline Technique for lipidomic analysis & Year of publication & EV types in the study \\
\hline Thin layer chromatography (TLC) & $\begin{array}{l}1987^{94} ; 1989^{95} ; 1995^{96} ; 2002^{97} ; 2004^{98} ; 2009^{99} ; 2010^{100} \\
2015^{101,102} ; 2017^{103}\end{array}$ & $\mathrm{sEV}^{94-96,98-103}, \mathrm{mEV} 96,98$ \\
\hline Liquid chromatography (HPLC, GLC, LC-CAD) & $2004 i^{98,104} 2010 i^{100} 2011 i^{.05} 2013 i^{.06} 2015 i^{.02} 2017^{107}$ & sEV98,100,102,104-106, mixed EV ${ }^{107}$ \\
\hline $\begin{array}{l}\text { MS-based techniques (ESI MS/MS; GC MS; LC } \\
\text { MS/MS) }\end{array}$ & $\begin{array}{l}2010^{100}, 2012^{108} ; 2013^{106,109-111} ; 2015^{112,113} ; 2016^{114-116} ; \\
2017^{117-119} ; 2018^{120-123}\end{array}$ & $\begin{array}{l}\mathrm{sEV}^{100,106,108-111,113,114,116,117,119-124}, \\
\mathrm{mEV}\end{array}$ \\
\hline
\end{tabular}

Table 3 Glycomic technologies used for EV analysis

\begin{tabular}{lll}
\hline Technique for glycomic analysis & Publications & Pros and cons \\
\hline Lectin-based microarrays & $125-129$ & Unbiased glycan analysis of carbohydrates on the surfaces of intact EVs \\
High resolution MS & Requires expensive equipment. Data analysis may be time consuming \\
\hline
\end{tabular}

\section{EV genomics}

Some of the EVs carry DNA that may range in size from 100 base pairs to several kilobase pairs ${ }^{39}$ or even fragments up to 2 million base pairs long ${ }^{40}$. EV-associated DNA may be single-stranded DNA, mitochondrial DNA, or double-stranded DNA ${ }^{39,41,42}$. The DNA content associated with EVs (termed EV-DNA) may be transported within the lumen of $\mathrm{EVs}^{39,40}$; however, recent studies have shown that, depending on the biological context, EVDNA can also be found attached to the outer surface of $\mathrm{EVs}^{43-46}$

Several studies have shown that EV-DNA spans sequences across all chromosomes of genomic DNA $(\mathrm{gDNA})^{39,40,47}$. Sequences of mitochondrial DNA (mtDNA) may or may not be present depending on the context and/or cell line ${ }^{39}$. Other studies have shown that selective sorting of specific DNA sequences may occur. For example, a study investigating different prostate cancer cell-derived EV subpopulations showed that different EVs carried different gDNA contents ${ }^{48}$. Another study that investigated the EVs of healthy individuals provided evidence of an uneven representation of the human genome and even detected EV-DNA of bacterial origin $^{46}$. Nevertheless, very little is currently known about the mechanisms of DNA packaging or selective sorting of DNA into EVs.

At present, the functional significance of EV-DNA is largely unknown. A recent study has shown that surfacebound EV-DNA plays a significant role in the binding of EVs to fibronectin ${ }^{45}$, an extracellular matrix glycoprotein that is of vital importance in processes associated with tumor progression ${ }^{49}$. Generally, surface-bound molecules are responsible for the binding of EVs to target cells or to the extracellular matrix ${ }^{50}$. Therefore, it is likely that exofacial EV-DNA may have some physiological significance for the recipient cells. Additionally, it has been shown that oncogenes can be transferred from donor to recipient cells; however, contradictory results have been reported regarding whether cancer cell-derived EV-DNA is functional in the recipient cells. In a study, the EVmediated spread of oncogenes was shown to promote disease progression in mice ${ }^{51}$. Another study showed that EVs containing oncogenic $\mathrm{H}$-ras failed to produce a permanent tumorigenic conversion of primary and immortalized fibroblasts ${ }^{52}$.

Several studies have shown that EV-DNA reflects the parental cell gDNA both qualitatively ${ }^{39,47,53-58}$ and quantitatively ${ }^{40,42}$. Therefore, the analysis of circulating EV-DNA may have substantial diagnostic potential. Moreover, the analysis of genomic mutations may prove to be superior to the analysis of the RNA transported by EVs, as DNA is intrinsically more stable than RNA.

\section{Systems biology approaches show relationships between genes involved in EV biogenesis and diseases}

Finally, it is possible to gain information about the role of EVs through a systems biology analysis of public transcriptomic and genomic data, as well as different types of biomedical data. Our goal was to determine the relationships between key genes involved in EV biogenesis and diseases using systems biology approaches. We investigated whether a selected group of the proteins from among those reported to play a role in the biogenesis or 


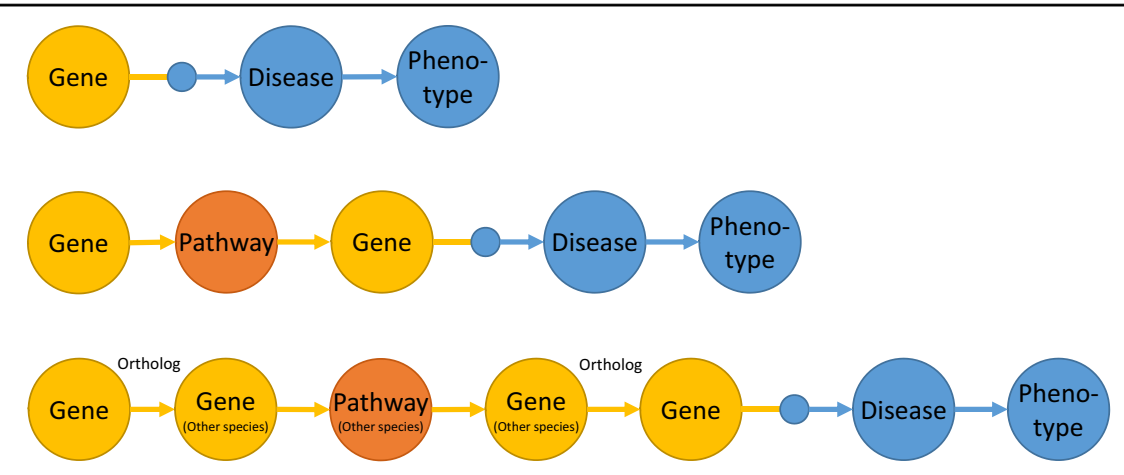

Fig. 1 Three different models used for prioritizing the associations of key EV genes with diseases. Top: A model that prioritizes diseases and phenotypes based on gene-disease associations known in the literature. Middle: This model predicts the associated diseases and phenotypes using molecular pathway associations. Bottom: This model predicts the associated diseases and phenotypes using orthologue molecular pathway

associations in other species

secretion of EVs were associated with phenotypes and were enriched in publicly available transcriptomic databases.

Based on the literature, without a claim of completeness, we have compiled lists of proteins that have been reported to play roles in the biogenesis (see Tables S1 and S3) and secretion of EVs (see Table S2). We defined five partly overlapping gene sets from among these lists, namely, genes involved in EV biogenesis and secretion, EV biogenesis, exosome biogenesis, microvesicle biogenesis, and exosome secretion. We used these sets as inputs for the different analyses. Of note, the term "exosomes" refers here to small (50-150 $\mathrm{nm}$ in diameter) EVs that originate from the multivesicular body, whereas the designation "microvesicles" is used for EVs shed from the plasma membrane that are usually of medium size $(100-1000 \mathrm{~nm}$ in diameter).

The Quantitative Semantic Fusion (QSF) System ${ }^{59}$ is an extensible framework that incorporates distinct annotated semantic types (also called entities) and links between them by integrating different data sources from the Linked Open Data world. The QSF System then enables the users to quantitatively prioritize a freely chosen entity based on evidence propagated from any other entity or possibly multiple entities through the connecting links (see Figure S1). Currently, the system contains genes, taxa, diseases, phenotypes, disease categories (UMLS semantic types and MeSH disease classes), pathways, substances, assays, cell lines, and the targets of the compounds. Links define associations between entities. For example, genes and pathways are connected with a link that represents gene-pathway associations. To enable cross-species information fusion, we also added gene orthologue links.

The most important gene-disease associations identified in this research are from the DisGeNet ${ }^{60}$ database. This database integrates many other sources of information (e.g., OMIM, GWAS Catalog, OrphaNet, Mouse Genome Database, and Rat Genome Database).

We constructed three different computation graphs that were used to detect known and predicted disease and phenotype associations (see Fig. 1). All three models can be used to answer the question of whether the genes involved in the biogenesis or secretion of EVs are functionally altered (for example, due to significant polymorphisms, mutations, or changes in the gene expression or the amount of protein produced), and, if so, which diseases are associated with these changes. This can also elucidate the pathomechanisms underlying the association between diseases or phenotypes and EVs.

The first model, based on gene-disease associations known in the literature (based on the data sources in the DisGeNet database), prioritizes the diseases and related phenotypes that can be linked to important genes relevant to EVs. In the second model, molecular pathway associations were used to expand the range of genes to include disease-associated genes that are in the same molecular pathways as the genes that are important for EVs. In the third model, we used the molecular pathway information from different species to predict the diseases associated with human genes the orthologues of which in other species are in the same molecular pathways as the orthologues of the human genes important for EVs.

We used the QSF System to quantitatively prioritize diseases and phenotypes that are associated with the five gene sets of genes known to be involved in the biogenesis and/or secretion of different types of EVs. First, we used a model that exploited the gene-disease associations already known in the literature. The top 20 diseases that are associated with genes that are involved either in the biogenesis or the secretion of EVs are shown in Table 4. The top 20 phenotypes are shown in Table S4. EV biogenesis genes are significantly associated with several diseases, including several tumors, such as mammary neoplasms 
Table 4 Diseases associated with different sets of key EV genes based on gene-disease associations known in the literature

\begin{tabular}{|c|c|c|c|c|c|c|c|c|c|c|}
\hline \multirow[t]{2}{*}{ Disease } & \multicolumn{2}{|c|}{$\begin{array}{l}\text { Extracellular vesicle } \\
\text { biogenesis and } \\
\text { secretion }\end{array}$} & \multicolumn{2}{|c|}{$\begin{array}{l}\text { Extracellular vesicle } \\
\text { biogenesis }\end{array}$} & \multicolumn{2}{|c|}{ Exosome biogenesis } & \multicolumn{2}{|c|}{$\begin{array}{l}\text { Microvesicle } \\
\text { biogenesis }\end{array}$} & \multicolumn{2}{|c|}{ Exosome secretion } \\
\hline & $\begin{array}{l}\text { Relevance } \\
\text { score }\end{array}$ & $P$-value & $\begin{array}{l}\text { Relevance } \\
\text { score }\end{array}$ & $P$-value & $\begin{array}{l}\text { Relevance } \\
\text { score }\end{array}$ & $P$-value & $\begin{array}{l}\text { Relevance } \\
\text { score }\end{array}$ & $P$-value & $\begin{array}{l}\text { Relevance } \\
\text { score }\end{array}$ & $P$-value \\
\hline Mammary neoplasms & 1.00 & 0.00 & 0.94 & 0.05 & 0.36 & 0.35 & 1.00 & 0.03 & 1.00 & 0.00 \\
\hline $\begin{array}{l}\text { Degenerative } \\
\text { polyarthritis }\end{array}$ & 0.72 & 0.00 & 0.55 & 0.00 & 0.34 & 0.04 & 0.41 & 0.03 & 1.00 & 0.00 \\
\hline Neoplasm invasiveness & 0.71 & 0.00 & 0.82 & 0.00 & 0.33 & 0.18 & 0.83 & 0.00 & 0.60 & 0.00 \\
\hline $\begin{array}{l}\text { Squamous cell } \\
\text { carcinoma }\end{array}$ & 0.66 & 0.00 & 0.37 & 0.02 & 0.04 & 0.27 & 0.52 & 0.00 & 0.84 & 0.00 \\
\hline Esophageal neoplasms & 0.60 & 0.00 & 0.56 & 0.02 & 0.01 & 0.22 & 0.83 & 0.00 & 0.60 & 0.00 \\
\hline $\begin{array}{l}\text { Diabetes mellitus, } \\
\text { experimental }\end{array}$ & 0.59 & 0.00 & 0.64 & 0.09 & & & 0.97 & 0.01 & 0.53 & 0.00 \\
\hline Neoplasm metastasis & 0.53 & 0.00 & 0.82 & 0.03 & 0.53 & 0.03 & 0.62 & 0.03 & 0.29 & 0.07 \\
\hline Liver carcinoma & 0.52 & 0.02 & 0.10 & 0.39 & 0.04 & 0.43 & 0.10 & 0.15 & 0.81 & 0.00 \\
\hline $\begin{array}{l}\text { Non-small cell lung } \\
\text { carcinoma }\end{array}$ & 0.49 & 0.00 & 0.29 & 0.12 & 0.00 & 0.91 & 0.45 & 0.03 & 0.61 & 0.00 \\
\hline Mouth neoplasms & 0.48 & 0.00 & & & & & & & 0.80 & 0.00 \\
\hline Melanoma & 0.47 & 0.02 & 0.68 & 0.02 & 0.39 & 0.10 & 0.55 & 0.02 & 0.30 & 0.05 \\
\hline $\begin{array}{l}\text { Animal mammary } \\
\text { neoplasms }\end{array}$ & 0.47 & 0.00 & & & & & & & 0.79 & 0.00 \\
\hline Juvenile-onset dystonia & 0.47 & 0.01 & & & & & & & 0.79 & 0.00 \\
\hline IGA glomerulonephritis & 0.44 & 0.07 & 1.00 & 0.00 & 1.00 & 0.05 & 0.28 & 0.32 & 0.20 & 0.12 \\
\hline $\begin{array}{l}\text { Mammary neoplasms, } \\
\text { experimental }\end{array}$ & 0.43 & 0.01 & 0.18 & 0.28 & & & 0.28 & 0.19 & 0.60 & 0.00 \\
\hline Alzheimer's disease & 0.43 & 0.06 & 0.32 & 0.15 & 0.01 & 0.74 & 0.48 & 0.06 & 0.49 & 0.02 \\
\hline Prostatic neoplasms & 0.42 & 0.11 & 0.07 & 0.65 & 0.01 & 0.77 & 0.10 & 0.30 & 0.65 & 0.00 \\
\hline Stomach neoplasms & 0.41 & 0.05 & 0.07 & 0.49 & 0.02 & 0.46 & 0.07 & 0.20 & 0.63 & 0.00 \\
\hline Adenocarcinoma & 0.40 & 0.01 & 0.33 & 0.08 & 0.00 & 0.75 & 0.48 & 0.02 & 0.43 & 0.01 \\
\hline $\begin{array}{l}\text { Myocardial reperfusion } \\
\text { injury }\end{array}$ & 0.39 & 0.02 & 0.64 & 0.00 & 0.22 & 0.17 & 0.97 & 0.00 & 0.20 & 0.11 \\
\hline
\end{tabular}

For each gene list (columns), the relevance score is the normalized relevance score computed by the first model (see Fig. 1). $P$-values were computed by permutation tests. The top 20 most relevant diseases are reported based on the gene list of EV biogenesis and secretion

(microvesicle biogenesis: $p=0.03$; Exosome secretion: $p<$ 0.01 ) and melanoma (microvesicle biogenesis: $p=0.02$ ); pathologic functions, such as neoplasm invasiveness (EV biogenesis and secretion: $p<0.01$ ) and neoplasm metastasis (EV biogenesis: $p=0.03$ ); and cardiovascular diseases, such as myocardial reperfusion injury (microvesicle biogenesis: $p<0.01)$. The most relevant phenotypes include frontotemporal dementia (exosome biogenesis: $p$ $<0.01$ ), lack of insight (exosome biogenesis: $p<0.01$ ), and autoimmune neutropenia (exosome secretion: $p=0.01$ ).
Pathway-mediated analysis (i.e., determining which diseases are associated with genes that participate in the same pathway as EV biogenesis genes) indicated possible associations of EVs with many common diseases (see Table S5 and Table S6), such as diabetes (microvesicle biogenesis: $p<0.01$ ), Alzheimer's disease (EV biogenesis and secretion: $p<0.01$ ), and obesity (microvesicle biogenesis: $p=0.02$ ). Cross-species pathway-mediated analysis indicated the possible association of EVs with several tumors (see Tables S7 and S8), such as mouth neoplasms 


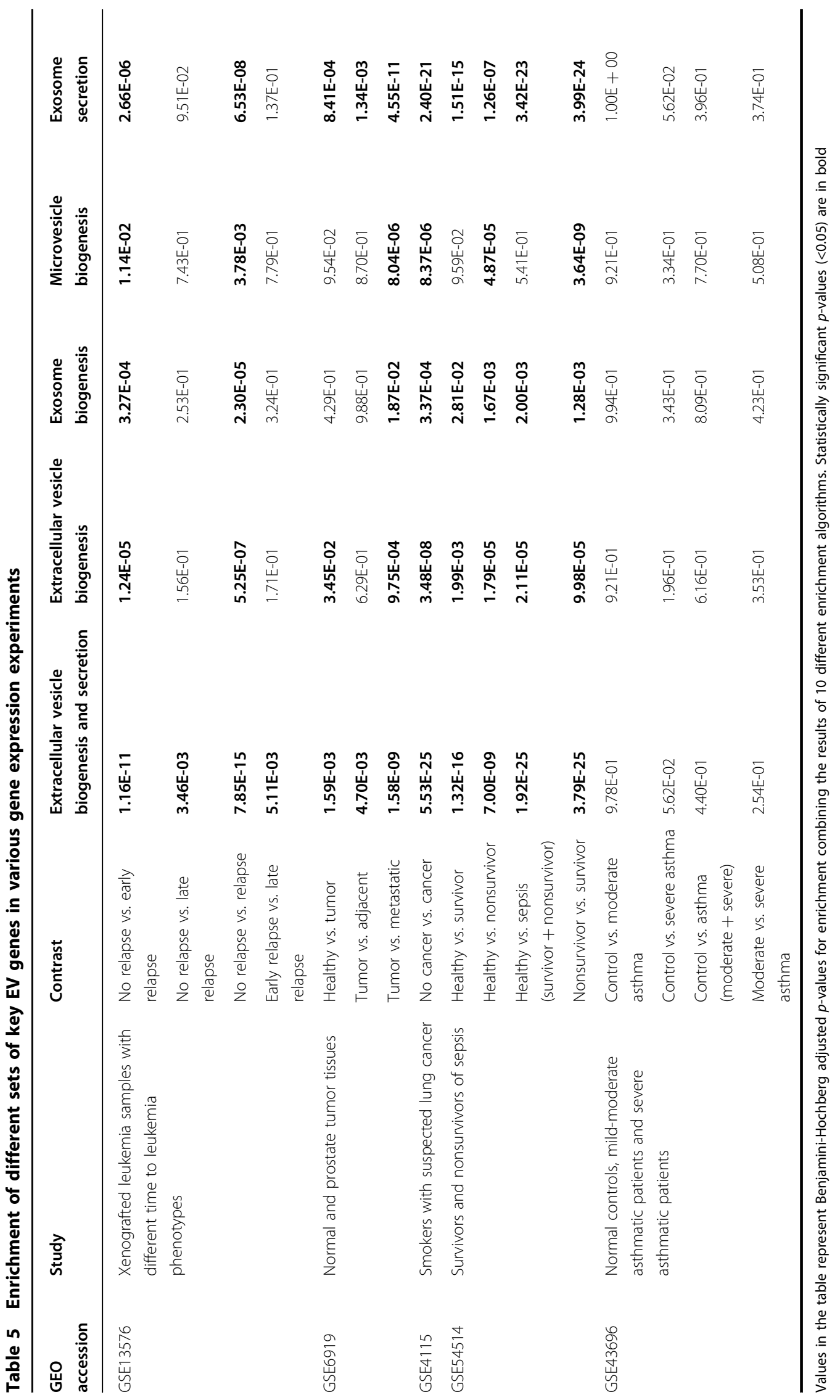


(exosome secretion: $p<0.01$ ) and tongue neoplasms (exosome secretion: $p<0.01$ ) and several other diseases and conditions.

Next, we downloaded and reanalyzed five large publicly available microarray data sets from the Gene Expression Omnibus (GEO) that represent various diseases (accessions: GSE13576, GSE6919, GSE4115, GSE54514, and GSE43696). Then, we computed the enrichment of the five key EV gene sets and all KEGG pathways in the various contrasts of the differential expression analyses. The statistical analyses were performed in $\mathrm{R}$ statistical language ${ }^{61}$. We used the limma ${ }^{62}$ and EGSEA ${ }^{63}$ packages for the microarray and enrichment analysis, respectively.

The Ensemble of Gene Set Enrichment Analysis (EGSEA) utilizes and combines the analysis results of many prominent gene set enrichment algorithms to calculate the collective significance score for a given gene set in the generally long lists of genes that arise from a differential expression analysis.

We reanalyzed five publicly available gene expression experiments using contrasts defined by the authors of these experiments, and then we computed the enrichment of the five key EV gene lists using the EGSEA method based on these contrasts (i.e., gene expression signatures relevant for a specific biological process).

The key EV gene sets were statistically significantly enriched in many of the analyzed contrasts (see Table 5).

Meyer et al. investigated the engraftment properties and impact on outcomes of 50 pediatric acute lymphoblastic leukemia samples transplanted into NOD/SCID mice ${ }^{64}$. They found that the time to the development of leukemia (i.e., weeks from transplant to overt leukemia) was strongly associated with the risk of early relapse. We found that the differentially expressed genes between the no relapse and the early relapse groups were significantly enriched for key EV genes as well.

$\mathrm{Yu}$ et al. performed a comprehensive gene expression analysis on 152 human samples, including prostate cancer tissues, prostate tissues adjacent to tumor, and organ donor prostate tissues, obtained from men of various ages ${ }^{65}$. The differentially expressed genes between the nonmetastatic tumor samples and the metastatic tumor samples were significantly enriched for all key EV gene sets.

Spira et al. compared gene expression data from smokers with lung cancer with samples from smokers without lung cancer ${ }^{66}$. This allowed them to generate a diagnostic gene expression profile that could distinguish between the two classes. We found that all EV gene sets were significantly enriched in the gene expression profile comparing smokers with and without lung cancer.

Parnell et al. performed gene expression profiling of whole blood to monitor immune dysfunction in critically ill septic patients ${ }^{67}$. We found that all gene expression signatures comparing healthy controls with sepsis survivors, healthy controls with nonsurvivors, and nonsurvivors with survivors were significantly enriched for EV genes.

Voraphani et al. compared the gene expression profiles of airway epithelial and bronchoalveolar lavage cells of healthy controls, mild-moderate asthmatic patients, and severe refractory asthmatic patients, respectively ${ }^{68}$. We found no enrichment in the different gene expression signatures.

Genes that have been reported to participate in the biogenesis or secretion of EVs are significantly associated with numerous common diseases, including different types of tumors and cardiovascular diseases, which further emphasizes the key role of EVs in human health and disease.

\section{Acknowledgements}

This work was supported by the National Scientific Research Program of Hungary (OTKA) grant no. 112872, 111958 and 120237, National Heart Program (NVKP_16-1-2016-0017) and the ÚNKP-18-3-IV-SE-14 New National Excellence Program of the Ministry of Human Capacities. Additional funding included VEKOP-2.3.2-16-2017-000002 and VEKOP-2.3.3-15-2017-00016, Higher Education Institutional Excellence Program - Therapeutic Development, STIA2017 and H2020-MSCA-ITN-2017-722148 TRAIN EV. The development of the Quantitative Semantic Fusion System was supported by the European Union's Horizon 2020 research and innovation program "Ageing with Elegans" (Grant agreement No 633589).

\section{Author details}

'Department of Genetics, Cell- and Immunobiology, Semmelweis University, Budapest, Hungary. ${ }^{2}$ MTA-SE Immune-Proteogenomics Extracellular Vesicle Research Group, Budapest, Hungary. ${ }^{3}$ Department of Measurement and Information Systems, Budapest University of Technology and Economics, Budapest, Hungary

\section{Conflict of interest}

The authors declare that they have no conflict of interest.

\section{Publisher's note}

Springer Nature remains neutral with regard to jurisdictional claims in published maps and institutional affiliations.

Supplementary information accompanies this paper at https://doi.org/ 10.1038/s12276-019-0226-2.

Received: 11 December 2018 Accepted: 12 December 2018. Published online: 15 March 2019

\footnotetext{
References

1. Mathivanan, S. \& Simpson, R. J. ExoCarta: a compendium of exosomal proteins and RNA. Proteomics 9, 4997-5000 (2009).

2. Kalra, H. et al. Vesiclepedia: a compendium for extracellular vesicles with continuous community annotation. PLoS Biol. 10, e1001450 (2012).

3. Pathan, M. et al. Vesiclepedia 2019: a compendium of RNA, proteins, lipids and metabolites in extracellular vesicles. Nucleic Acids Res. 47, D516-D519 (2019).

4. Kim, D.-K. et al. EVpedia: an integrated database of high-throughput data for systemic analyses of extracellular vesicles. J. Extracell. Vesicles 2, 20384 (2013).

5. Kim, D.-K. et al. EVpedia: a community web portal for extracellular vesicles research. Bioinformatics 31, 933-939 (2015).

6. Cheung, K-H. et al. Extending gene ontology in the context of extracellular RNA and vesicle communication. J. Biomed. Semant. 7, 19 (2016).
} 
7. Keerthikumar S., Gangoda L., Gho Y. S. \& Mathivanan S. Bioinformatics Tools for Extracellular Vesicles Research. In: Exosomes and Microvesicles. Methods in molecular biology (eds Hill, A. \& Clifton, N. J.) pp 189-196 (Humana Press, New York, 2017).

8. Pathan, M. et al. A novel community driven software for functional enrichment analysis of extracellular vesicles data. J. Extracell. Vesicles 6, 1321455 (2017).

9. Sódar, B. W. et al. Best practice of identification and proteomic analysis of extracellular vesicles in human health and disease. Expert. Rev. Proteom. 14, 1073-1090 (2017).

10. Pocsfalvi, G. et al. Mass spectrometry of extracellular vesicles. Mass. Spectrom. Rev. 35, 3-21 (2016).

11. Wang, N. et al. Circulating exosomes contain protein biomarkers of metastatic non-small-cell lung cancer. Cancer Sci. 109, 1701-1709 (2018).

12. Sun, Y. et al. Systematic comparison of exosomal proteomes from human saliva and serum for the detection of lung cancer. Anal. Chim. Acta 982, 84-95 (2017).

13. Gonzalez-Calero, L. et al. Urinary exosomes reveal protein signatures in hypertensive patients with albuminuria. Oncotarget 8, 44217-44231 (2017).

14. Arbelaiz, A. et al. Serum extracellular vesicles contain protein biomarkers for primary sclerosing cholangitis and cholangiocarcinoma. Hepatology 66 , 1125-1143 (2017).

15. $\mathrm{Xu}, \mathrm{Y}$. et al. Exosomal proteome analysis of human plasma to monitor sepsis progression. Biochem. Biophys. Res. Commun. 499, 856-861 (2018).

16. Cvjetkovic, A. et al. Detailed analysis of protein topology of extracellular vesicles-evidence of unconventional membrane protein orientation. Sci. Rep. 6, 36338 (2016).

17. Amorim, M. G. et al. A total transcriptome profiling method for plasmaderived extracellular vesicles: applications for liquid biopsies. Sci. Rep. 7, 14395 (2017).

18. Buschmann, D. et al. Evaluation of serum extracellular vesicle isolation methods for profiling miRNAs by next-generation sequencing. J. Extracell. Vesicles 7, 1481321 (2018).

19. Rekker, $\mathrm{K}$. et al. Comparison of serum exosome isolation methods for microRNA profiling. Clin. Biochem. 47, 135-138 (2014).

20. Li, M. et al. Analysis of the RNA content of the exosomes derived from blood serum and urine and its potential as biomarkers. Philos. Trans. R. Soc. Lond. B. Biol. Sci. 369, 20130502-20130502 (2014).

21. Huang, $X$. et al. Characterization of human plasma-derived exosomal RNAs by deep sequencing. BMC Genom. 14, 319 (2013).

22. Miranda, K. C. et al. Massively parallel sequencing of human urinary exosome/microvesicle RNA reveals a predominance of non-coding RNA. PLOS One 9, e96094 (2014).

23. Yagi, Y. et al. Next-generation sequencing-based small RNA profiling of cerebrospinal fluid exosomes. Neurosci. Lett. 636, 48-57 (2017).

24. Ogawa, Y., Taketomi, Y., Murakami, M., Tsujimoto, M. \& Yanoshita, R. Smal RNA transcriptomes of two types of exosomes in human whole saliva determined by next generation sequencing. Biol. Pharm. Bull. 36, 66-75 (2013).

25. Palanisamy, $\mathrm{V}$. et al. Nanostructural and transcriptomic analyses of human saliva derived exosomes. PLoS One 5, e8577 (2010).

26. Hunter, M. P. et al. Detection of microRNA expression in human peripheral blood microvesicles. PLoS One 3, e3694 (2008).

27. Driedonks, T. A. P. et al. Immune stimuli shape the small non-coding transcriptome of extracellular vesicles released by dendritic cells. Cell Mol. Life Sci. 75, 3857-3875 (2018).

28. Juzenas, S. et al. A comprehensive, cell specific microRNA catalogue of human peripheral blood. Nucleic Acids Res. 45, 9290-9301 (2017).

29. Hubal, M. J. et al. Circulating adipocyte-derived exosomal MicroRNAs associated with decreased insulin resistance after gastric bypass. Obes. (Silver Spring). 25, 102-110 (2017).

30. Palomo, L. et al. Considerations for applying metabolomics to the analysis of extracellular vesicles. Front. Immunol. 5, 651 (2014).

31. Clos-Garcia, M. et al. Metabolic alterations in urine extracellular vesicles are associated to prostate cancer pathogenesis and progression. J. Extracell. Vesicles 7, 1470442 (2018).

32. Puhka, M. et al. Metabolomic profiling of extracellular vesicles and alternative normalization methods reveal enriched metabolites and strategies to study prostate cancer-related changes. Theranostics 7, 3824-3841 (2017).

33. Casal, E., Palomo, L., Cabrera, D. \& Falcon-Perez, J. M. A novel sensitive method to measure catechol-O-methyltransferase activity unravels the presence of this activity in extracellular vesicles released by rat hepatocytes. Front. Pharmacol. 7, 501 (2016).

34. Altadill, T. et al. Enabling metabolomics based biomarker discovery studies using molecular phenotyping of exosome-like vesicles. PLoS One 11, e0151339 (2016)

35. Iraci, N. et al. Extracellular vesicles are independent metabolic units with asparaginase activity. Nat. Chem. Biol. 13, 951-955 (2017).

36. Royo, F. et al. Hepatocyte-secreted extracellular vesicles modify blood metabolome and endothelial function by an arginase-dependent mechanism. Sci. Rep. 7, 42798 (2017).

37. Royo, F. et al. Metabolically active extracellular vesicles released from hepatocytes under drug-induced liver-damaging conditions modify serum metabolome and might affect different pathophysiological processes. Eur. J. Pharm. Sci. 98, 51-57 (2017).

38. Lopes-Rodrigues, $\mathrm{V}$. et al. Identification of the metabolic alterations associated with the multidrug resistant phenotype in cancer and their intercellular transfer mediated by extracellular vesicles. Sci. Rep. 7, 44541 (2017).

39. Thakur, B. K. et al. Double-stranded DNA in exosomes: a novel biomarker in cancer detection. Cell Res. 24, 766-769 (2014).

40. Vagner, $T$. et al. Large extracellular vesicles carry most of the tumour DNA circulating in prostate cancer patient plasma. J. Extracell. Vesicles 7, 1505403 (2018).

41. Guescini, M., Genedani, S., Stocchi, V. \& Agnati, L. F. Astrocytes and glioblastoma cells release exosomes carrying mtDNA. J. Neural Transm. 117, 1-4 (2010).

42. Balaj, L. et al. Tumour microvesicles contain retrotransposon elements and amplified oncogene sequences. Nat. Commun. 2, 180 (2011).

43. Shelke G., Jang S. C., Yin Y., Lässer C., Lötvall J. Human mast cells release extracellular vesicle-associated DNA. Matters (Zürich), https://doi.org/ 10.19185/matters.201602000034 (2016).

44. Fischer, S. et al. Indication of horizontal DNA gene transfer by extracellular vesicles. PLoS One 11, e0163665 (2016).

45. Németh, A. et al. Antibiotic-induced release of small extracellular vesicles (exosomes) with surface-associated DNA. Sci. Rep. 7, 8202 (2017).

46. Grigor'eva, A. E. et al. Exosomes in tears of healthy individuals: isolation, identification, and characterization. Biochem Suppl. Ser. B Biomed. Chem. 10, 165-172 (2016).

47. Kahlert, $C$. et al. Identification of double-stranded genomic DNA spanning all chromosomes with mutated KRAS and p53 DNA in the serum exosomes of patients with pancreatic cancer. J. Biol. Chem. 289, 3869-3875 (2014).

48. Lázaro-Ibáñez, E. et al. Different gDNA content in the subpopulations of prostate cancer extracellular vesicles: apoptotic bodies, microvesicles, and exosomes. Prostate 74, 1379-1390 (2014).

49. Wang, J. P. \& Hielscher, A. Fibronectin: how its aberrant expression in tumors may improve therapeutic targeting. J. Cancer 8, 674-682 (2017).

50. Yáñez-Mó, M. et al. Biological properties of extracellular vesicles and their physiological functions. J. Extracell. Vesicles 4, 1-60 (2015).

51. Cai, J. et al. Transferred BCR/ABL DNA from K562 extracellular vesicles causes chronic myeloid leukemia in immunodeficient mice. PLoS One 9, e105200 (2014).

52. Lee, T. H. et al. Barriers to horizontal cell transformation by extracellular vesicles containing oncogenic H-ras. Oncotarget 7, 51991-52002 (2016).

53. San Lucas, F. A. et al. Minimally invasive genomic and transcriptomic profiling of visceral cancers by next-generation sequencing of circulating exosomes. Ann. Oncol. 27, 635-641 (2016).

54. Allenson, $\mathrm{K}$. et al. High prevalence of mutant KRAS in circulating exosomederived DNA from early stage pancreatic cancer patients. Ann. Oncol. 28, $\mathrm{mdx004}$ (2017).

55. Möhrmann, L. et al. Liquid biopsies using plasma exosomal nucleic acids and plasma cell-free DNA compared with clinical outcomes of patients with advanced cancers. Clin. Cancer Res. 24, 181-188 (2018).

56. Yang, S. et al. Detection of mutant KRAS and TP53 DNA in circulating exosomes from healthy individuals and patients with pancreatic cancer. Cancer Biol. Ther. 18, 158-165 (2017).

57. Castellanos-Rizaldos, E. et al. Exosome-based detection of EGFR T790M in plasma from non-small cell lung cancer patients. Clin. Cancer Res. 24, 2944-2950 (2018)

58. García-Romero, N. et al. DNA sequences within glioma-derived extracellular vesicles can cross the intact blood-brain barrier and be detected in peripheral blood of patients. Oncotarget 8, 1416-1428 (2017). 
59. Gezsi A., Bruncsics B., Guta G. \& Antal P. Constructing a Quantitative Fusion Layer over the Semantic Level for Scalable Inference. In: Bioinformatics and Biomedical Engineering (eds Rojas, I. \& Ortuño, F.) IWBBIO 2018, pp 41-53 (Springer, Cham, 2018).

60. Queralt-Rosinach, N., Piñero, J., Bravo, À., Sanz, F. \& Furlong, L. I. DisGeNETRDF: harnessing the innovative power of the Semantic Web to explore the genetic basis of diseases. Bioinformatics 32, 2236-2238 (2016).

61. R. Core Team. R: A Language and Environment for Statistical Computing. R Foundation for Statistical Computing, Vienna, Austria (2018). https://www. R-project.org/.

62. Ritchie, M. E. et al. Limma powers differential expression analyses for RNAsequencing and microarray studies. Nucleic Acids Res. 43, e47 (2015)

63. Alhamdoosh, $M$. et al. Combining multiple tools outperforms individual methods in gene set enrichment analyses. Bioinformatics 33 414-424 (2017).

64. Meyer, L. H. et al. Early relapse in ALL is identified by time to leukemia in $\mathrm{NOD/SCID}$ mice and is characterized by a gene signature involving survival pathways. Cancer Cell. 19, 206-217 (2011).

65. Yu, Y. P. et al. Gene expression alterations in prostate cancer predicting tumor aggression and preceding development of malignancy. J. Clin. Oncol. 22 2790-2799 (2004).

66. Spira, A. et al. Airway epithelial gene expression in the diagnostic evaluation of smokers with suspect lung cancer. Nat. Med. 13, 361-366 (2007).

67. Parnell, G. P. et al. Identifying key regulatory genes in the whole blood of septic patients to monitor underlying immune dysfunctions. Shock 40, 166-174 (2013).

68. Voraphani, N. et al. An airway epithelial iNOS-DUOX2-thyroid peroxidase metabolome drives Th1/Th2 nitrative stress in human severe asthma. Mucosal Immunol. 7, 1175-1185 (2014).

69. $L V$, $L$. et al. RNA profiling analysis of the serum exosomes derived from patients with active and latent mycobacterium tuberculosis infection. Front. Microbiol. 8, 1051 (2017).

70. Jia, H.-L. et al. MicroRNA expression profile in exosome discriminates extremely severe infections from mild infections for hand, foot and mouth disease. Bmc. Infect. Dis. 14, 506 (2014).

71. Ghai, V. et al. Genome-wide profiling of urinary extracellular vesicle microRNAs associated with diabetic nephropathy in type 1 diabetes. Kidney Int Rep. 3, 555-572 (2018).

72. Santovito, D. et al. Plasma exosome microRNA profiling unravels a new potential modulator of adiponectin pathway in diabetes: effect of glycemic control. J. Clin. Endocrinol. Metab. 99, E1681-E1685 (2014).

73. Matsumoto, S. et al. Circulating p53-responsive microRNAs are predictive indicators of heart failure after acute myocardial infarction. Circ. Res. 113 322-326 (2013).

74. Royo, F. et al. Transcriptomic profiling of urine extracellular vesicles reveals alterations of CDH3 in prostate cancer. Oncotarget 7, 6835-6846 (2016).

75. Liu, X. et al. Metastatic breast cancer cells overexpress and secrete miR-218 to regulate type I collagen deposition by osteoblasts. Breast Cancer Res. 20, 127 (2018).

76. Conley, A. et al. High-throughput sequencing of two populations of extracellular vesicles provides an mRNA signature that can be detected in the circulation of breast cancer patients. RNA Biol. 14, 305-316 (2017).

77. Ostenfeld, M. S. et al. miRNA profiling of circulating EpCAM(+) extracellular vesicles: promising biomarkers of colorectal cancer. J. Extracell. Vesicles 5, 31488 (2016).

78. Lutgendorf, S. K. et al. Biobehavioral modulation of the exosome transcriptome in ovarian carcinoma. Cancer 124, 580-586 (2018).

79. Taylor, D. D. \& Gercel-Taylor, C. MicroRNA signatures of tumor-derived exosomes as diagnostic biomarkers of ovarian cancer. Gynecol. Oncol. 110, 13-21 (2008).

80. Vaksman, O., Tropé, C., Davidson, B. \& Reich, R. Exosome-derived miRNAs and ovarian carcinoma progression. Carcinogenesis 35, 2113-2120 (2014).

81. Zhou, $X$. et al. A six-microRNA panel in plasma was identified as a potential biomarker for lung adenocarcinoma diagnosis. Oncotarget 8, 6513-6525 (2017).

82. Rabinowits, G., Gerçel-Taylor, C., Day, J. M., Taylor, D. D. \& Kloecker, G. H. Exosomal microRNA: a diagnostic marker for lung cancer. Clin. Lung. Cancer 10, 42-46 (2009).

83. Ragusa, M. et al. miRNA profiling in vitreous humor, vitreal exosomes and serum from uveal melanoma patients: pathological and diagnostic implications. Cancer Biol. Ther. 16, 1387-1396 (2015).
84. Bao, Q. et al. Extracellular vesicle RNA sequencing reveals dramatic transcriptomic alterations between metastatic and primary osteosarcoma in a liquid biopsy approach. Ann. Surg. Oncol. 25, 2642-2651 (2018).

85. Noerholm, M. et al. RNA expression patterns in serum microvesicles from patients with glioblastoma multiforme and controls. BMC Cancer 12, 22 (2012).

86. McKeever, P. M. et al. MicroRNA expression levels are altered in the cerebrospinal fluid of patients with young-onset Alzheimer's disease. Mol. Neurobiol. 55, 8826-8841 (2018).

87. Kimura, $\mathrm{K}$. et al. Circulating exosomes suppress the induction of regulatory T cells via let-7i in multiple sclerosis. Nat. Commun. 9, 17 (2018).

88. Selmaj, I. et al. Global exosome transcriptome profiling reveals biomarkers for multiple sclerosis. Ann. Neurol. 81, 703-717 (2017).

89. Sukma Dewi, I. et al. Exosomal miR-142-3p is increased during cardiac allograft rejection and augments vascular permeability through downregulation of endothelial RAB11FIP2 expression. Cardiovasc. Res. 113, 440-452 (2017).

90. Gregson, A. L. et al. Altered exosomal RNA profiles in bronchoalveolar lavage from lung transplants with acute rejection. Am. J. Respir. Crit. Care. Med. 192, 1490-1503 (2015).

91. Abu-Halima, M. et al. Altered micro-ribonucleic acid expression profiles of extracellular microvesicles in the seminal plasma of patients with oligoasthenozoospermia. Fertil. Steril. 106, 1061-1069.e3 (2016).

92. Gildea, J. J., Carlson, J. M., Schoeffel, C. D., Carey, R. M. \& Felder, R. A. Urinary exosome miRNome analysis and its applications to salt sensitivity of blood pressure. Clin. Biochem. 46, 1131-1134 (2013)

93. Belleannée, C., Légaré, C., Calvo, E., Thimon, V. \& Sullivan, R. microRNA signature is altered in both human epididymis and seminal microvesicles following vasectomy. Hum. Reprod. 28, 1455-1467 (2013).

94. Johnstone, R. M., Adam, M., Hammond, J. R., Orr, L. \& Turbide, C. Vesicle formation during reticulocyte maturation. Association of plasma membrane activities with released vesicles (exosomes). J. Biol. Chem. 262, 9412-9420 (1987).

95. Vidal, M., Sainte-Marie, J., Philippot, J. R. \& Bienvenue, A. Asymmetric distribution of phospholipids in the membrane of vesicles released during in vitro maturation of guinea pig reticulocytes: evidence precluding a role for "aminophospholipid translocase". J. Cell Physiol. 140, 455-462 (1989).

96. Fourcade, O. et al. Secretory phospholipase A2 generates the novel lipid mediator lysophosphatidic acid in membrane microvesicles shed from activated cells. Cell 80, 919-927 (1995).

97. Weerheim, A. M., Kolb, A. M., Sturk, A. \& Nieuwland, R. Phospholipid composition of cell-derived microparticles determined by one-dimensional highperformance thin-layer chromatography. Anal. Biochem. 302, 191-198 (2002).

98. Laulagnier, K et al. Mast cell- and dendritic cell-derived exosomes display specific lipid composition and an unusual membrane organization. Biochem. J. 380, 161-171 (2004).

99. Parolini, I. et al. Microenvironmental $\mathrm{pH}$ is a key factor for exosome traffic in tumor cells. J. Biol. Chem. 284, 34211-34222 (2009).

100. Subra, C. et al. Exosomes account for vesicle-mediated transcellular transport of activatable phospholipases and prostaglandins. J. Lipid Res. 51, 2105-2120 (2010).

101. Bourderioux, M. et al. A new workflow for proteomic analysis of urinary exosomes and assessment in cystinuria patients. J. Proteome Res. 14, 567-577 (2015).

102. Roier, S. et al. A basis for vaccine development: comparative characterization of Haemophilus influenzae outer membrane vesicles. Int. J. Med. Microbiol. 305, 298-309 (2015).

103. Blans, K. et al. Pellet-free isolation of human and bovine milk extracellular vesicles by size-exclusion chromatography. J. Extracell. Vesicles 6, 1294340 (2017).

104. Laulagnier, K. et al. PLD2 is enriched on exosomes and its activity is correlated to the release of exosomes. FEBS Lett. 572, 11-14 (2004).

105. Carayon, K. et al. Proteolipidic composition of exosomes changes during reticulocyte maturation. J. Biol. Chem. 286, 34426-34439 (2011).

106. Brouwers, J. F. et al. Distinct lipid compositions of two types of human prostasomes. Proteomics 13, 1660-1666 (2013).

107. Ribeiro, D. et al. Extracellular vesicles from human pancreatic islets suppress human islet amyloid polypeptide amyloid formation. Proc. Natl Acad. Sci. USA 114, 11127-11132 (2017)

108. Vallejo, M. C. et al. Lipidomic analysis of extracellular vesicles from the pathogenic phase of Paracoccidioides brasiliensis. PLoS One 7, e39463 (2012). 
109. Baig, S. et al. Lipidomic analysis of human placental syncytiotrophoblast microvesicles in adverse pregnancy outcomes. Placenta 34, 436-442 (2013).

110. Rappa, G., Mercapide, J., Anzanello, F., Pope, R. M. \& Lorico, A. Biochemical and biological characterization of exosomes containing prominin-1/CD133. Mol. Cancer 12, 62 (2013).

111. Llorente, A. et al. Molecular lipidomics of exosomes released by PC-3 prostate cancer cells. Biochim. Biophys. Acta 1831, 1302-1309 (2013).

112. Pienimaeki-Roemer, A. et al. Lipidomic and proteomic characterization of platelet extracellular vesicle subfractions from senescent platelets. Transfusion 55, 507-521 (2015)

113. Lydic, T. A. et al. Rapid and comprehensive 'shotgun' lipidome profiling of colorectal cancer cell derived exosomes. Methods 87, 83-95 (2015).

114. Haraszti, R. A. et al. High-resolution proteomic and lipidomic analysis of exosomes and microvesicles from different cell sources. J. Extracell. Vesicles $\mathbf{5}$, 32570 (2016)

115. $\mathrm{Hu}, \mathrm{Q}$. et al. Lipid profile of platelets and platelet-derived microparticles in ovarian cancer. BBA Clin. 6, 76-81 (2016).

116. Ouyang, $Y$. et al. Isolation of human trophoblastic extracellular vesicles and characterization of their cargo and antiviral activity. Placenta 47, 86-95 (2016).

117. Durcin, M. et al. Characterisation of adipocyte-derived extracellular vesicle subtypes identifies distinct protein and lipid signatures for large and small extracellular vesicles. J. Extracell. Vesicles 6, 1305677 (2017).

118. Braig, D. et al. Transitional changes in the CRP structure lead to the exposure of proinflammatory binding sites. Nat. Commun. 8, 14188 (2017).

119. Dang, V. D., Jella, K. K., Ragheb, R. R. T., Denslow, N. D. \& Alli, A. A. Lipidomic and proteomic analysis of exosomes from mouse cortical collecting duct cells. FASEB J. 31, 5399-5408 (2017).

120. Hough, K. P. et al. Unique lipid signatures of extracellular vesicles from the airways of asthmatics. Sci. Rep. 8, 10340 (2018).

121. Jeon, J. et al. Comparative lipidomic profiling of the human commensal bacterium Propionibacterium acnes and its extracellular vesicles. RSC Adv. 8 , 15241-15247 (2018).

122. Downs, C. A., Dang, V. D., Johnson, N. M., Denslow, N. D. \& Alli, A. A. Hydrogen peroxide stimulates exosomal cathepsin $\mathrm{B}$ regulation of the receptor for advanced glycation end-products (RAGE). J. Cell Biochem. 119, 599-606 (2018)
123. Miranda, A. M. et al. Neuronal lysosomal dysfunction releases exosomes harboring APP C-terminal fragments and unique lipid signatures. Nat. Commun. 9, 291 (2018)

124. Chao, O. S. et al. The HDAC6 inhibitor tubacin induces release of CD133 +extracellular vesicles from cancer cells. J. Cell. Biochem. 118, 4414-4424 (2017).

125. Krishnamoorthy, L., Bess, J. W., Preston, A. B., Nagashima, K. \& Mahal, L. K. HIV-1 and microvesicles from $T$ cells share a common glycome, arguing for a common origin. Nat. Chem. Biol. 5, 244-250 (2009).

126. Batista, B. S., Eng, W. S., Pilobello, K. T., Hendricks-Muñoz, K. D. \& Mahal, L. K. Identification of a conserved glycan signature for microvesicles. J. Proteome Res. 10, 4624-4633 (2011).

127. Shimoda, A., Tahara, Y., Sawada, S.-I., Sasaki, Y. \& Akiyoshi, K. Glycan profiling analysis using evanescent-field fluorescence-assisted lectin array: Importance of sugar recognition for cellular uptake of exosomes from mesenchymal stem cells. Biochem. Biophys. Res. Commun. 491, 701-707 (2017).

128. Pilobello, K. T., Krishnamoorthy, L., Slawek, D. \& Mahal, L. K. Development of a lectin microarray for the rapid analysis of protein glycopatterns. Chembiochem 6, 985-989 (2005).

129. Echevarria, J. et al. Microarray-based identification of lectins for the purification of human urinary extracellular vesicles directly from urine samples. Chembiochem 15, 1621-1626 (2014).

130. Escrevente, C. et al. Sialoglycoproteins and N-glycans from secreted exosomes of ovarian carcinoma cells. PLoS One 8, e78631 (2013).

131. Gomes, J. et al. Extracellular vesicles from ovarian carcinoma cells display specific glycosignatures. Biomolecules 5, 1741-1761 (2015).

132. Nyalwidhe, J. O. et al. Increased bisecting N-acetylglucosamine and decreased branched chain glycans of $\mathrm{N}$-linked glycoproteins in expressed prostatic secretions associated with prostate cancer progression. Proteom. Clin. Appl. 7, 677-689 (2013).

133. Staubach, S., Schadewaldt, P., Wendel, U., Nohroudi, K. \& Hanisch, F.-G. Differential glycomics of epithelial membrane glycoproteins from urinary exovesicles reveals shifts toward complex-type $\mathrm{N}$-glycosylation in classical galactosemia. J. Proteome Res. 11, 906-916 (2012)

134. Zhang, $H$. et al. Identification of distinct nanoparticles and subsets of extracellular vesicles by asymmetric flow field-flow fractionation. Nat. Cell Biol. 20, 332-343 (2018). 